\title{
Wide dynamic range homodyne interferometry method and its application for piezoactuator displacement measurements
}

\author{
José Henrique Galeti, ${ }^{1}$ Paula Lalucci Berton, ${ }^{1}$ Cláudio Kitano, ${ }^{1}$ Ricardo Tokio Higuti, ${ }^{1, \star}$ \\ Ronny Calixto Carbonari, ${ }^{2}$ and Emílio Carlos Nelli Silva ${ }^{3}$ \\ 'Universidade Estadual Paulista—UNESP, Department of Electrical Engineering, Avenida Brasil 56, \\ 15385-000 Ilha Solteira, SP, Brazil
}

${ }^{2}$ Federal University of ABC, Centro de Engenharia, Modelagem e Ciências Sociais Aplicadas, Rua Santa Adélia, 166, 09210-170 Santo André, SP, Brazil

${ }^{3}$ Escola Politécnica da Universidade de São Paulo_EPUSP, Department of Mechatronics and Mechanical Systems Engineering, Av. Prof. Mello Moraes, 2231, 05508-030 São Paulo, Brazil

${ }^{*}$ Corresponding author: tokio@ dee.feis.unesp.br

Received 9 July 2013; revised 3 September 2013; accepted 3 September 2013;

posted 4 September 2013 (Doc. ID 193094); published 26 September 2013

\begin{abstract}
Multiactuated piezoelectric flextensional actuators (MAPFAs) is a fast-growing technology in development, with a wide range of applications in precision mechanics and nanotechnology. In turn, optical interferometry is an adequate technique to measure nano/micro-displacements and to characterize these MAPFAs. In this work, an efficient method for homodyne phase detection, based on a well-known Bessel functions recurrence relation, is developed, providing practical applications with a high dynamic range. Fading and electronic noise are taken into account in the analysis. An important advantage of the method is that, for each measurement, only a limited number of frequencies in the magnitude spectrum of the photodetected signal are used, without the need to know the phase spectrum. The dynamic range for phase demodulation is from 0.2 to $100 \pi \mathrm{rad}$ (or $10 \mathrm{~nm}$ to $16 \mu \mathrm{m}$ for displacement, using $632.8 \mathrm{~nm}$ wavelength). The upper range can be easily expanded by adapting the electronic system to the signal characteristics. By using this interferometric technique, a new XY nanopositioner MAPFA prototype is tested in terms of linearity, displacement frequency response, and coupling rate. () 2013 Optical Society of America

OCIS codes: (120.3180) Interferometry; (120.0120) Instrumentation, measurement, and metrology; (120.5050) Phase measurement; (120.7280) Vibration analysis.

http://dx.doi.org/10.1364/AO.52.006919
\end{abstract}

\section{Introduction}

The main parameters that characterize actuator performance are displacement range, force, bandwidth, size, weight, and power consumption. In general, most actuators exhibit good performance in some of these characteristics but are inadequate in others.

$1559-128 X / 13 / 286919-12 \$ 15.00 / 0$

(C) 2013 Optical Society of America
Examples of widely used actuators are the piezoelectric, magnetostrictive, microelectromechanical system (MEMS)-based electrostatic, MEMS-based electromagnetic, MEMS-based thermal, etc. [1]. Piezoelectric actuators are ubiquitous tools in a wide variety of applications, particularly those involving micro/nanomanipulation. These actuators have excellent bandwidth and can generate mechanical forces in a compact design with small power consumption. However, they provide relatively small 
displacement ranges [1] and, consequently, the raw piezoceramic displacements should be amplified in order to operate in multiscales.

Conventional manipulator systems, based on pins and guides, present backlash and stiction of the revolute joints, as well as geometric and dimensional errors, preventing their application in micro/ nanomanipulation [2]. Therefore, different mechanisms must be developed to overcome this problem. One of the best solutions is to use flexure-based mechanisms, where conventional kinematic parts are replaced by flexure hinges. Novel multiactuated piezoelectric flextensional actuator (MAPFA) families have been developed using the topology optimization method, which combines an optimization algorithm and the finite element method (FEM) in order to find the optimum topology of the mechanical parts of the system $[3,4]$. The actuators discussed in this paper are examples of MAPFAs, consisting essentially of multiflexible structures actuated by two or more piezoceramics that should generate different output displacements and forces at different points of the specific domain and at different directions.

These types of mechanisms have a number of advantages related to backlash, zero friction, and negligible hysteresis [5]. Such piezoelectric positioners can be used in atomic force microscopes for positioning the sample, in scanning tunneling microscopes for positioning the probe [6] , lens and mirrors positioners for laser interferometers [7], in cellular manipulation [8], in microsurgery [9], and others.

However, despite being essential in MAPFA design (as XY nanopositioners, for example), compliant mechanisms produce an undesired effect, which is the cross talk between $\mathrm{X}$ and $\mathrm{Y}$ displacements $[3, \underline{4}]$. That is, the displacement in a direction results in a spurious displacement in the perpendicular direction. This means that special care should be taken to solve this problem when designing ultraprecision nanopositioners. Consequently, measurements of displacement of such structures are of great importance to verify analytical and FEM models, to access the material and device properties, to detect defects, and to evaluate performance. These data can also be used to provide an additional optimization of the device design.

In this article, one MAPFA device, developed by using the topology optimization method $[3,4]$, is experimentally analyzed to evaluate some of its characteristics such as movement output and coupling displacement. An elementary homodyne Michelson interferometer is used, where one of the beams impinges on the actuator vibrating surface. A major advantage of optical interferometry is its capability to measure physical quantities with a very high sensitivity. However, interferometry is also invariably corrupted by large quasi-static phase random drifts due to external environmental disturbances. This phenomenon is well known as signal fading $[10,11]$.
A large number of phase-demodulation techniques have been described in the literature to detect the induced phase shift under fading: active homodyne [12], heterodyne [13], phase quadrature [14], and synthetic heterodyne [15], among many others $[10,11]$. There is general agreement that heterodyne techniques, with picometer resolution, are useful when the displacement exceeds an interference fringe, while homodyne techniques are often employed when the displacement does not exceed the fringe spacing [16]. However, homodyne interferometers still have interesting characteristics, such as ease of use, low cost, and small periodic error level, which are much more pronounced in heterodyne interferometers [17]. Moreover, with the availability of inexpensive processing power, some computational signal-processing complexity is often preferable to recover the optical path length from the measured photocurrent, instead of additional optics and electronics that are required by other techniques. Because of this, a new passive homodyne-detection method, based on the analysis of the photodetected signal spectrum, is presented. Essentially the method is used to recover the value of the modulation index $(x)$, which is then related to the desired displacement amplitude $(\Delta L)$.

Well-established methods based on spectrum analysis, such as the $J_{1} \ldots J_{4}[\underline{18}]$ and $J_{1} \ldots J_{6}[\underline{19}]$, for example, have the advantages of being simple, direct, self-consistent, and immune to fading. However, they have reduced dynamic ranges, between 0.2 and $3.8 \mathrm{rad}$ and between 0.035 and $6.1 \mathrm{rad}$, respectively. A method described by Pernick in 1973 [20] was among the first proposed to detect the value of the modulation index $x$ from the spectral components of the photodetected signal. This method was not specially named, as happened with the $J_{1} \max , J_{1}$ null, $J_{1} / J_{2}, J_{1} / J_{3}[\underline{21}], J_{1} \ldots J_{4}[\underline{18}]$, and $J_{1} \ldots J_{6}$ [19] methods, for example. In this text, it will be referred to as the Pernick method, in honor of its creator.

According to the Pernick method, the photodetector output at three harmonics, selected according to a chosen $n$ value $(1,2,3, \ldots)$, are used to calculate the dynamic phase-modulation index. The Pernick method is elegant and mathematically consistent, but, curiously, to the authors' knowledge, no practical implementation of the method has been published in the literature, as happened, for example, with the $J_{1} \ldots J_{4}$ method, published 16 years later.

Furthermore, in principle, the Pernick method should allow the exact calculation of $x$ using indistinctly $n=2$ or $n=3$, for example, and also should not depend on the current value of $x$, which might take any value between zero and infinity. That is, any values of $x$ could be measured independently of the selected value of $n$. However, as noted by the authors (and as will be shown in the next sections), there may be severe limitations when taking into account the highly restrictive effects of electronic noise and signal fading, which were not considered by Pernick. 
It is not the sole objective of this work just to revisit the method originally proposed by Pernick and test it in experiments. The main contribution of this paper is the proposal of an efficient algorithm with a large dynamic range and computational robustness for displacement measurement. In view of the severe problems intrinsic to the Pernick method, which will be discussed below, an algorithm to properly select the integer value of $n$ is proposed, according to the modulation index value $(x)$ to be measured.

Therefore, by taking advantage of a well-known Bessel function recurrence relation and simple computational resources, a method for automatic measurements of dynamic phase, subject to effects of electronic noise and environmental drifts, is presented. Modulation indexes $x$ ranging from $0.2 \mathrm{rad}$ up to $100 \pi$ rad can be measured using the proposed method. The method is direct (no transcendental equations), is self-consistent (no self-calibration needed), does not present phase ambiguity problems (due to oscillatory behavior of Bessel functions), is immune to fading, and has a large dynamic range, wide enough for practical measurements of MAPFA displacements. Another advantage of the proposed approach is that only the harmonic magnitudes are necessary, regardless of their phases. Consequently, it does not need algebraic sign correction algorithms, as the modified $J_{1} \ldots J_{4}$ method [22].

This paper is divided into six sections, including this introduction. In Section 2, the MAPFA used in this work is presented. In Section 3 , the original Pernick method is examined, pointing out its limitations when electronic noise and fading are considered. The modified Pernick method is proposed in Section 4 , and some computational simulations are presented to confirm its efficiency. Experiments about the MAPFA linearity, frequency response, and coupling rate are presented in Section 5 . Conclusions are presented in Section $\underline{6}$.

\section{Multiactuated Piezoelectric Flextensional Actuators}

The design of a MAPFA is relatively complex, as it involves several piezoceramics, with a large number of actuating displacements and mainly due to the displacement cross coupling, which is quite critical. The cross coupling is undesirable and imposes a limitation in the device efficiency and control. A comprehensive discussion on the design of these mechanisms and compliant nanopositioners by using the topology optimization method can be found in $[3,4,23]$.

The $\overline{X Y}$ nanopositioner shown in Fig. 1 is a MAPFA with two piezoceramics (PZT-5A, American Piezoceramics) glued with epoxy resin to an aluminum structure and has been designed to have maximum displacements $(\Delta x$ and $\Delta y)$ at its right corner. The displacement in $\mathrm{X}(\mathrm{Y})$ direction, for example, is generated by exciting the upper (lower) piezoceramic, while the displacement in $\mathrm{Y}(\mathrm{X})$ direction is the cross-coupled one. The aluminum structure is

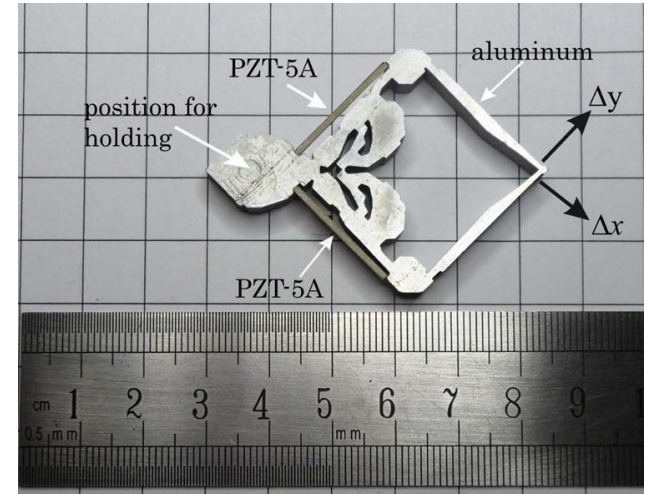

Fig. 1. XY nanopositioner prototype.

fabricated by wire electron discharge machining technique, and the two piezoceramics have dimensions $20 \mathrm{~mm} \times 6 \mathrm{~mm} \times 1 \mathrm{~mm}$ in directions 1,2 , and 3 , respectively. The electrodes are deposited on plane $1-2$, and the piezoceramic is polarized in direction 3. Reflective tape (3M, Scotchlite 7610, formed by micro glass beads with $60 \mu \mathrm{m}$ diameter) was adhered at the measurement points of interest in order to reflect a significant portion of the optical beam of the sensor arm of the interferometer.

A quantitative measure of the cross talk between $\mathrm{X}$ and $\mathrm{Y}$ displacements in an XY nanopositioner can be given in terms of percentage by $S_{x y}=\Delta y_{s} / \Delta x$ [23], where $\Delta x$ is the desired displacement in the $\mathrm{X}$ direction (the generated displacement), and $\Delta y_{s}$ is the spurious coupled displacement in the $\mathrm{Y}$ direction. Consequently, it is important to decrease $S_{x y}$ in the XY nanopositioner design step $[3,4]$.

It should be emphasized that the $\overline{X Y}$ nanopositioners designed and manufactured by the authors should operate in a static or quasi-static regime in practical applications, below the first resonance frequencies $[3,4,23]$. As the first resonance frequency is not a parameter that is taken into account in the present MAPFAs computational design, interferometry plays an important role in the measurement of frequency response, as well as linearity, bandwidth, and cross coupling.

\section{Pernick Method}

Laser metrology is the technique of choice for highaccuracy displacement measurements. Two-beam interferometers are often used in practical metrology systems because they can ensure displacement measurement traceability [24]. Among the different available configurations, the Michelson interferometer is adequate for many practical applications [25]. By considering a sinusoidal input phase shift described by $\Delta \phi(t)=x \sin \left(\omega_{s} t+\phi_{s}\right)$, where the frequency $\omega_{s}(\mathrm{rad} / \mathrm{s})$ is known, $x(\mathrm{rad})$ is the unknown modulation index, and $\phi_{s}$ (rad) is a random initial phase, the photodetected voltage at the output of a homodyne Michelson interferometer can be expanded in Fourier series, resulting in [18] 


$$
\begin{aligned}
v(t)= & A\left(\frac{1}{2}+F\left\{\frac{Q}{2} J_{0}(x)\right.\right. \\
& -P \sum_{i=1}^{\infty} J_{2 i-1}(x) \sin \left[(2 i-1)\left(\omega_{s} t+\phi_{s}\right)\right] \\
& \left.\left.+Q \sum_{i=1}^{\infty} J_{2 i}(x) \cos \left[2 i\left(\omega_{s} t+\phi_{s}\right)\right]\right\}\right),
\end{aligned}
$$

where $J_{i}(x)$ are Bessel functions of the first kind, and order $i=1,2,3, \ldots, Q=\cos \phi_{0}$, and $P=\sin \phi_{0}$ depend on the random phase $\phi_{0}$. The proportionality constant $A$ depends on the optical source intensity, the photodetector responsivity, and the signal conditioning circuit gain. Factor $F(0 \leq F \leq 1)$ is related to the interference fringe visibility and $\phi_{0}$ is a static phase (in principle). Temperature fluctuations, air turbulence, and mechanical vibrations at the interferometer site can cause large variations in the phase $\phi_{0}$. For these reasons, in this work, $\phi_{0}$ will be denoted by $\phi_{0}(t)$, which corresponds to a random and slow phase component. The odd and even harmonics of $v(t)$ have amplitudes

$$
V_{2 i-1}(x)=A F P J_{2 i-1}(x)
$$

and

$$
V_{2 i}(x)=A F Q J_{2 i}(x),
$$

respectively. Usually the information corresponding to the DC term $J_{0}(x)$ is not used because it suffers large influence from electronic and ambient noise [26]. Pernick established the following expression to obtain the modulation index $x$ from the harmonics amplitudes [20]:

$$
x^{2}=\frac{4 n(n+1)(n+2) J_{n+1}(x)}{(n+2) J_{n-1}(x)+2(n+1) J_{n+1}(x)+n J_{n+3}(x)},
$$

which can be easily obtained from the well-known recurrence relation $J_{n+1}(x)=2 n J_{n}(x) / x-J_{n-1}(x)$.

The substitution of Eqs. (2a) and (2b) in (3) is the essence of the method, which proposes to estimate the modulation index from the harmonic components of $v(t)$ :

$\left(x^{\prime}\right)^{2}=\frac{4 n(n+1)(n+2) V_{n+1}(x)}{(n+2) V_{n-1}(x)+2(n+1) V_{n+1}(x)+n V_{n+3}(x)}$,

where $x^{\prime}$ is the estimated (or measured), and $x$ is the expected values of the modulation indexes. In Eq. (4), the $A F P$ or $A F Q$ factors are canceled, which means that in this technique there is no dependence on random phase $\phi_{0}(t)$, fringe visibility, photodetector responsivity, or optical source oscillations. Consequently, in principle, the method is direct, selfconsistent, and immune to fading. By choosing an odd value of $n$ in Eq. (4), it is necessary to use three even harmonics. For example, for $n=1: V_{0}, V_{2}$, and $V_{4}$; for $n=3: V_{2}, V_{4}$, and $V_{6}$, and so on. By choosing an even value of $n$, three odd harmonics are used (for $n=2: V_{1}, V_{3}$, and $V_{5}$; for $n=4: V_{3}, V_{5}$, and $V_{7}$, and so on). According to Pernick, the user should look for the best value of $n$ according to the experimental circumstances and suggests that the value $n=1$ should be avoided, once it would be difficult to distinguish $V_{0}(x)$ from the DC component $(A / 2)$ in $v(t)$ given in Eq. (1).

As discussed below, it is not possible to use a single value of $n$ in Eq. (4) to estimate the value of $x$ in a wide range. Furthermore, the method has a resolution limit, which is the minimum finite modulation index $x$ that can be measured, which depends on the random noise. In order to consider the effect of electronic noise on the photodetected signal, a deterministic formulation proposed in [27] will be followed, which is valid for noise voltages that vary with frequency $f$ according to $1 / f^{m}$, where $m$ is a positive integer that depends on the noise type. Using this approach, the noise-free voltage components in Eqs. (2a $)$ and (마) are substituted by

$$
V_{2 i-1}(x)=A F\left[P J_{2 i-1}(x)+K /(2 i-1)^{m}\right]
$$

and

$$
V_{2 i}(x)=A F\left[Q J_{2 i}(x)+K /(2 i)^{m}\right],
$$

where $K$ is a noise factor defined by $K=\Delta V_{1} / A F$, and $\Delta V_{1}$ is the noise voltage measured at the fundamental frequency. For the particular value of $m=1$ used in [27], the noise is generated by semiconductor junctions in optical and electronic components (laser, photodetector, amplifier, acquisition system, etc.). This kind of noise will be employed in this paper only for comparison with other authors, once the experimental measurements show, in the frequency bands explored in this work, that white noise $(m=0)$ predominates.

For example, by substituting Eqs. (5a) and (5b) in $(\underline{4})$, using $n=2, m=1, \phi_{0}(t)=\pi / 4 \overline{\mathrm{rad}}$, and $K=$ 0.0011, which is the same as those used in [19] [this value of $K$ is conservative because in this work the authors have measured values of the order of 0.0004 for white noise $(m=0)$ ], the curve of $x^{\prime}$ as a function of $x$ is presented in Fig. 2(a). The case for $n=3$ is shown in Fig. 2(b). The relative percent error, $\Delta x_{r}=$ $100\left|x^{\prime}-x\right| / x$, is also shown in the figures. Moduli and phases of $V_{i}(x)$ were taken into account in the calculation of the results shown in these figures.

Ideally, Eq. (4) should lead to the relation $x^{\prime}(x)=x$ in the absence of noise. However, when $x \ll 1 \mathrm{rad}$, $V_{n}(x)$ have very small magnitudes for $n>1$, which can be below the electronic noise level in the system. Consequently, there is a lower limit in resolution. Otherwise, even without considering the effect of noise, for $x=6.38 \mathrm{rad}$ the numerator and denominator of Eq. () become zero, originating a singularity of 

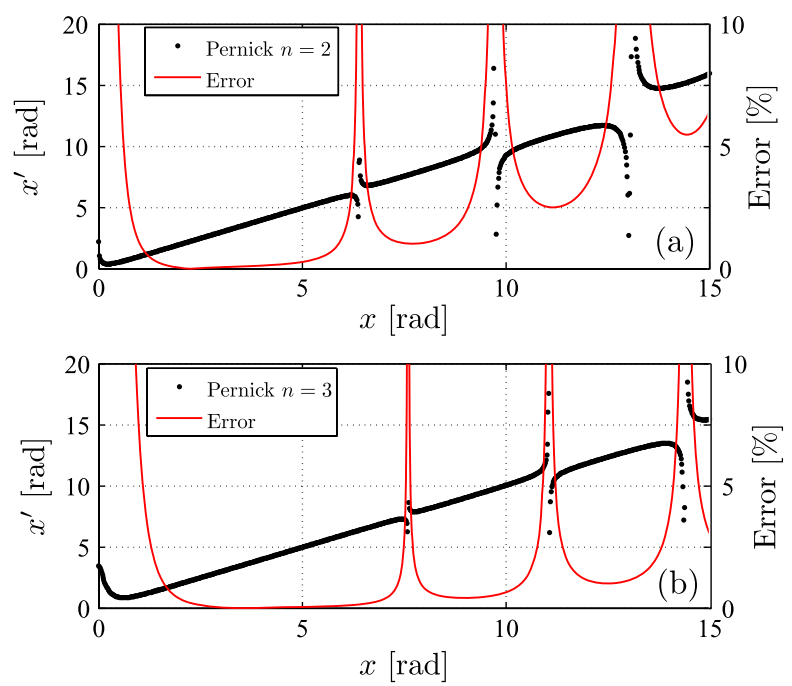

Fig. 2. Relation between $x^{\prime}$ and $x$ for the Pernick method with (a) $n=2$ and (b) $n=3$. The percent relative error $\Delta x$ is also represented.

the type $0 / 0$ at this point. This would generate only a single point discontinuity in the curve $x^{\prime}(x)$, and its consequences would not be significant. However, as can be seen in Fig. 2(a), this discontinuity also depends on noise, and the error increases to intolerable levels in a finite width around $x=6.38 \mathrm{rad}$. Other $0 / 0$ singularities exist for $n=2$ at 9.76, 13.01, $16.22 \mathrm{rad}$, and so on. Consequently, the Pernick method for $n=2$ has limited dynamic range, between 0.1790 and $5.9476 \mathrm{rad}$, approximately, according to the discussion in Section 4. There is a similar behavior for $n=3$, which has a dynamic range between 0.4274 and 7.3328 rad.

An additional problem related to the Pernick method refers to the algebraic signs of Bessel functions, which should be corrected when the magnitude (or modulus) of the spectral components are used in Eqs. (2)-(4), without considering the phase. This was also a problem encountered by Jin et al. [22] for the $J_{1} \ldots J_{4}$ method, which operated only up to $3.83 \mathrm{rad}$. At this value of $x, J_{1}(x)$ becomes negative for the first time, and by using only the magnitude information, the dynamic range of this method is limited, between 0.2 (due to noise) and $3.83 \mathrm{rad}$, approximately [19]. To solve this problem and indefinitely extend the dynamic range of the $J_{1} \ldots J_{4}$ method, Jin et al. proposed the modified $J_{1} \ldots J_{4}$ method [22], in which the knowledge of the initial phase $\phi_{s}$ is necessary, in order to correct the spectral components algebraic signs in Eqs. (2a) and (2b) for higher values of $x$. However, the arbitrary phase $\phi_{s}$ depends on the initial instant of acquisition and sampling of the interferometric signal. In order to take these matters into account in the analysis, a complicated algorithm to correct the algebraic signs was proposed, which can expand the dynamic range up to approximately $5.1 \mathrm{rad}$ only. This is because the authors of the modified $J_{1} \ldots J_{4}$ method did not take into account the effect of electronic noise. Actually, around $x=5.1 \mathrm{rad}$, for the
$J_{1} \ldots J_{4}$ method (modified or no), there is a $0 / 0$ singularity [19] that, also considering electronic noise, results in a finite region where the error increases to intolerable values.

Besides these two problems in the Pernick method, there is another one, if, at the measurement instant, the random phase assumes a value that is an integer multiple of $\pi / 2 \mathrm{rad}$. That is, if $\phi_{0}(t)=p \pi / 2 \mathrm{rad}$, $p=0, \pm 1, \pm 2, \pm 3, \ldots$, then $P=0$ or $Q=0$ in Eq. (2), resulting in 0/0 singularities in Eq. (4) for $n$ even and odd, respectively. This random value of $\phi_{0}(t)$ should rarely occur and would have no more consequences than generating one or other point out of the linearity curve $x^{\prime}(x)$. Furthermore, this specific measurement could be repeated later and the problematic data discarded. However, the experiments could take longer because the rate of change of $\phi_{0}(t)$ is slow, and the authors of this work have been observing that these singularity points occur often in open-loop homodyne interferometers and in normal laboratory conditions.

In order to complete the discussion about the performance of the Pernick method, the relative percent error as a function of modulation index $x$ and phase $\phi_{0}$ for $n=2, m=1, K=0.0011,0 \leq x \leq 15 \mathrm{rad}$, and $0 \leq \phi_{0} \leq 2 \pi \mathrm{rad}$ are shown in Fig. 3. This tridimensional graph is called a bathtubs graph, for obvious reasons. The value of $x^{\prime}$ was obtained from the moduli and phases of the harmonics using Eq. (4). The error values were truncated to $10 \%$ in order to make the interpretation of the results easier. The percentage error shown in Fig. 2(a) corresponds to the particular case of this graph when $\phi_{0}(t)=\pi / 4 \mathrm{rad}$. The error can exceed $100 \%$ in several regions: close to $x=0$, close to values of $x$ that cause $0 / 0$ singularities, and close to the instants when $\phi_{0}(t)=p \pi / 2 \mathrm{rad}$ (in this case, for $0, \pi$ and $2 \pi \mathrm{rad}$ ). The occurrence of many bathtubs in this error graph means there are deficiencies in the method. Similar graphs would be obtained for other values of $n$ in Eq. (4).

By observing Fig. 2 again, one can conclude that the Pernick method, when applied with a single value of $n$, results in a limited dynamic range, as happens with the $J_{1} \ldots J$, modified $J_{1} \ldots J_{4}$, and

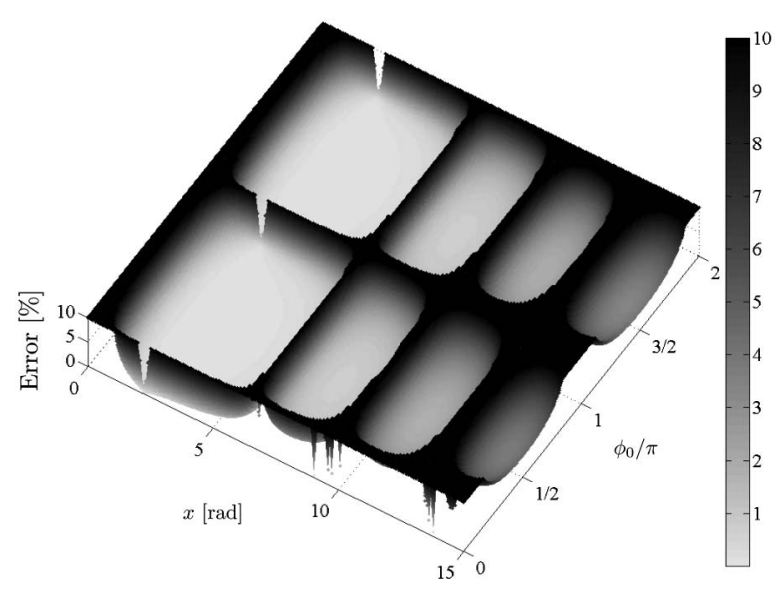

Fig. 3. Bathtubs graph for the Pernick method using $n=2$. 
$J_{1} \ldots J$ methods, for example [19]. However, unlike the other methods, the Pernick method can be adapted to be used for any integer value of $n$, each one with its own dynamic range. For a determined value of $n$, there are large errors in the vicinities of $x=0$ and near the first $0 / 0$ singularity, but in between these values the error is extremely small. Consequently, each value of $n$ corresponds to a particular dynamic range. These dynamic ranges are complementary, which give rise to the proposed method, with large global dynamic range.

\section{4. $n$-Commuted Pernick Method}

In this work, a generalization of the Pernick method is proposed, which is the denominated $n$-Commuted Pernick method ( $n$-CPM). The objective is to establish a standard and computationally simple procedure to estimate values of $x$ as high as $100 \pi \mathrm{rad}$ (or higher), by selecting an adequate value of $n$ in Eq. (4), according to the information contained in the photodetected signal $v(t)$ itself. The challenge is to do it when there is fading, which is manifested by the low-frequency random phase $\phi_{0}(t)$, and working only with the magnitude of $V_{n}(x)$, without considering the phase.

In principle, the selection of an adequate value of $n$ can be accomplished by more than one way, but the one that is proposed, because of its simplicity and efficiency, is:

Proposition: Given a value of $x$ and considering $\phi_{0}(t)$ a random signal, then, if $k$ is the order of the higher magnitude harmonic $\left|V_{k}\right|$ of the photodetected signal, a good choice for $n$ in Eq. (4) will be $n=k+1$, which means that the harmonics of interest will be of order $n-1=k, n+1=k+2$ and $n+3=k+4$. This will be valid always that $k \geq 3$. There are two ambiguity cases for $k=1$ and $k=2$. When $k=1$ and $\left|V_{5} / V_{1}\right|<0.6$, then $n=2$; otherwise, if $\left|V_{5} / V_{1}\right| \geq 0.6$, then $n=4$. When $k=2$ and $\left|V_{6} / V_{2}\right|<0.6$, then $n=3$; otherwise, if $\left|V_{6} / V_{2}\right| \geq$ 0.6 , then $n=5$.

This is a sufficient condition to choose an adequate value of $n$, but it is not the only one. For example, another possibility is to choose $n=k-1$ (with other thresholds for the ambiguity cases when $k=1$ or 2 ), but in this case it is necessary to correct the algebraic sign of the Bessel functions. Additionally, some harmonic components may have very low magnitudes within the range of $x$ associated to $n$, resulting in problems due to noise.

In this work, the dynamic range is defined as in $[19,27]$; it is lower-limited by the minimum detectable phase shift (MDPS) and upper-limited when the error exceeds $0.05 \mathrm{rad}$. The MDPS was defined in order to estimate the resolution of the method in the presence of noise and is the value of $x$ when the absolute error satisfies $\Delta x=\left|x^{\prime}-x\right|=x$ in the presence of noise. Following this procedure, the dynamic ranges for each value of $n$ (up to 10) in Eq. (4) are obtained and presented in Table 1. Modulus and phase of $V_{n}(x)$ are taken into account to
Table 1. Dynamic Range as a Function of $\boldsymbol{n}^{\mathrm{a}}$

\begin{tabular}{lcc}
\hline$n$ & Lower Limit [rad] & Upper Limit [rad] \\
\hline 2 & 0.1790 & 5.9476 \\
3 & 0.4274 & 7.3328 \\
4 & 0.7618 & 8.5824 \\
5 & 1.1616 & 9.7818 \\
6 & 1.6113 & 10.9534 \\
7 & 2.0997 & 12.1064 \\
8 & 2.6187 & 13.2461 \\
9 & 3.1622 & 14.3752 \\
10 & 3.7524 & 15.4956 \\
\hline
\end{tabular}

${ }^{a}$ Magnitude and phase of the harmonic $V_{i}(x)$ are taken into account.

obtain the dynamic ranges. The different ranges have superpositions that are suitable for establishing a robust method in the presence of noise and fading in practical cases. Although this analysis has been made only for a few values of $n$, it can be easily extended to values as large as $n=100$, for example.

Before explaining the proposition, it is instructive to revise the behavior of the Bessel functions $J_{i}(x)$ and the harmonics $V_{i}(x)$ in Eq. (2) in the absence of noise. The Bessel functions $J_{i}(x)$ are decaying oscillatory functions of $x$ and the first maximum of $J_{1}(x)$, which occurs at $x=1.8 \mathrm{rad}$, is higher than the first maximum of $J_{2}(x)$ at $x=3.2 \mathrm{rad}$, which is higher than the first maximum of $J_{3}(x)$ at $x=$ $4.2 \mathrm{rad}$, and so on. The same happens for the second maxima and so on. However, in Eq. (4), the photodetected voltage magnitudes $V_{n}$ are proportional to these Bessel functions multiplied by the random functions $P=\sin \phi_{0}(t)$ (odd harmonics) or $Q=$ $\cos \phi_{0}(t)$ (even harmonics). Then, if at measurement instant $t_{0}$ the phase is $\phi_{0}\left(t_{0}\right)=\pi / 8 \mathrm{rad}$, for example, then the first maximum of $\left|V_{1}(x)\right|$ is $0.2225 A F$, which is now smaller than the first maximum of $\left|V_{2}(x)\right|$, which is $0.4482 A F$. Consequently, the maximum harmonic magnitude $\left|V_{k}\right|$ for a determined value of $x$ depends on the values of $J_{n}(x)$ and $\phi_{0}(t)$ at the measurement instant. That is, for a given value of $x$ the higher harmonic of $v(t)$ in Eq. (1) can be randomly odd or even, depending on the current value of $x$ and $\phi_{0}(t)$. Furthermore, as the same factor $\cos \phi_{0}(t)$ multiplies all the even harmonics and $\sin \phi_{0}(t)$ multiplies all the odd harmonics, for a determined value of $\phi_{0}(t)$, all even harmonics in the spectrum of $v(t)$ increase (decrease) while all odd harmonics decrease (increase) simultaneously and in the same proportion. As a consequence, it is convenient to analyze the behavior of the envelope of maxima as a function of $x$ for several values of $\phi_{0}(t)$. This analysis will be done for several ranges of $\phi_{0}(t)$ and, for clarity, only for $0<x<18 \mathrm{rad}$ in the cases studied here, but can be easily extended for higher values of $x$.

\section{A. Range of $\phi_{0}$ (t) between $\pi / 2 \pm 0.3156 \pi \mathrm{rad}$ and between $3 \pi / 2 \pm 0.3156 \pi \mathrm{rad}$}

As the proposition uses the information relative to the harmonics with maximum magnitude, it is 
convenient to know the interval of $x$ where each harmonic has maximum magnitude. This is shown as the envelope of maxima in Fig. 4: the magnitudes of maxima of Eq. (2), normalized by $A F$, for $(0<x<$ 12) $\mathrm{rad}$ and three values of $\phi_{0}(t): \pi / 2,0.3634 \pi$, and $0.3156 \pi \mathrm{rad}$.

For the values of $\phi_{0}(t)$ considered in this subsection, the following observations can be made: as $P=$ $\sin \phi_{0}(t) \sim 1$ (in modulus) and $Q=\cos \phi_{0}(t) \sim 0$, the even harmonics are always smaller than the odd harmonics; the length of the range of $x$ corresponding to each harmonic $k$ which has maximum envelope, remains constant and is independent of $\phi_{0}(t)$; as the value of $x$ increases from zero, there is a region where $\left|V_{1}(x)\right|$ is maximum for $0 \leq x \leq 3.0558 \mathrm{rad}$; then $\left|V_{3}(x)\right|$ is maximum from 3.0558 to $5.1363 \mathrm{rad}$; then $\left|V_{1}(x)\right|$ is maximum again, from 5.1363 to 5.6164; then $\left|V_{5}(x)\right|$ for $5.6164 \leq x \leq 7.5019 \mathrm{rad}$. The authors have observed that for higher values of $x$ after this point, the sequence of maxima are related to functions $\left|V_{7}(x)\right|,\left|V_{9}(x)\right|,\left|V_{11}(x)\right|$, etc. This was verified up to $x=100 \pi \mathrm{rad}$, but is possibly valid until infinity. Consequently, there is only one ambiguity case, related to function $\left|V_{1}(x)\right|$ (for $k=1$ ), which is maximum at two disjoint intervals. This ambiguity case is provided in the proposition and will be further discussed in Subsection 4.D.

In order to analyze other intervals for $\phi_{0}(t)$, a function that indicates which harmonic envelope of order $k$ is larger for each pair of $\left(x, \phi_{0}\right)$ was generated for $0 \leq x \leq 10 \mathrm{rad}$ (without loss of generality) and $0 \leq \phi_{0}(t) \leq \pi / 2 \mathrm{rad}$, as shown in Fig. 5 . Actually, this is obtained from the top view of a three-dimensional surface of $\left|V_{k}\right|$ versus $x$ and $\phi_{0}(t)$, and the values of $k$ where $\left|V_{k}\right|$ are maximum and are shown in gray colors. Due to the symmetry of the problem, only the first quadrant is considered.

The analyzed interval in this subsection corresponds to the portion between $\phi_{0}(t)=0.3156 \pi$ and

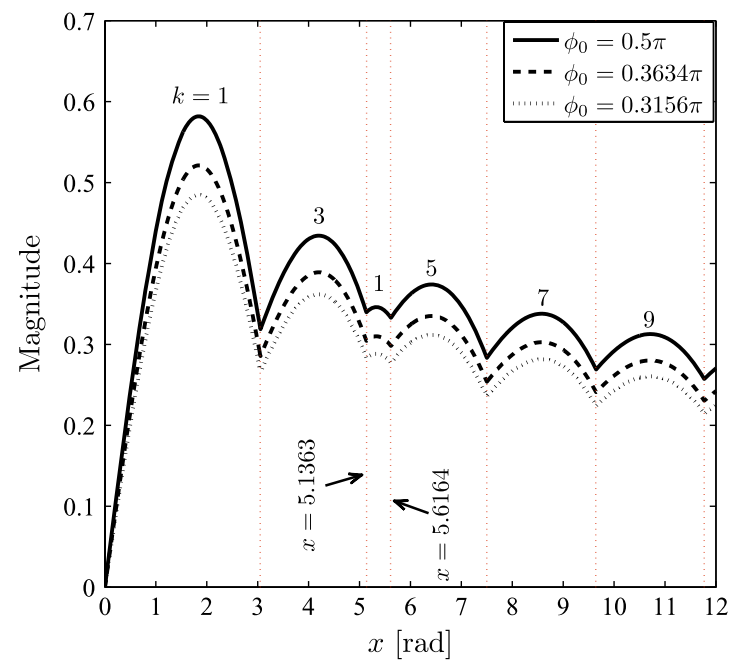

Fig. 4. Envelope of maxima $\left|V_{k}(x)\right| / A F$ and three values of $\phi_{0}(t)$ : $0.3156 \pi, 0.3634 \pi$, and $\pi / 2 \mathrm{rad}$. The numbers above the peaks indicate the order of the harmonic with a larger magnitude in that interval.

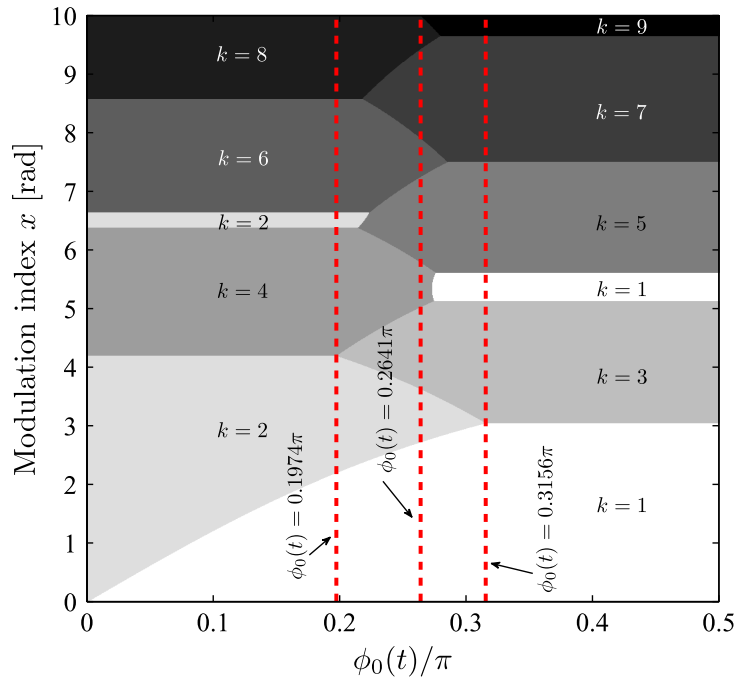

Fig. 5. Function that indicates which harmonic envelope of order $k$ is larger for each pair of $\left(x, \phi_{0}\right)$.

$\pi / 2$ in Fig. 5 . It is now clearer from the figure that in this interval of $\phi_{0}(t)$, the envelope of maxima are related to odd-order harmonics only, and the corresponding ranges of $x$ in which each harmonic is larger do not change with $\phi_{0}(t)$ for each value of $k$.

Now it is useful to compare the intervals where these functions are maximum with the intervals related to the dynamic ranges for each value of $n$ (or $k$ ). By using the information from Fig. 5 and Table 1 , Fig. 6(a) is obtained, where the regions in white represent the $x$ intervals corresponding to the maximum magnitude harmonic of order $k$ (odd), in the range of $\phi_{0}(t)$ considered in this subsection. The regions in gray (which contain the white strips) represent the dynamic ranges for each value of $n$, as in Table 1 [considering modulus and phase of
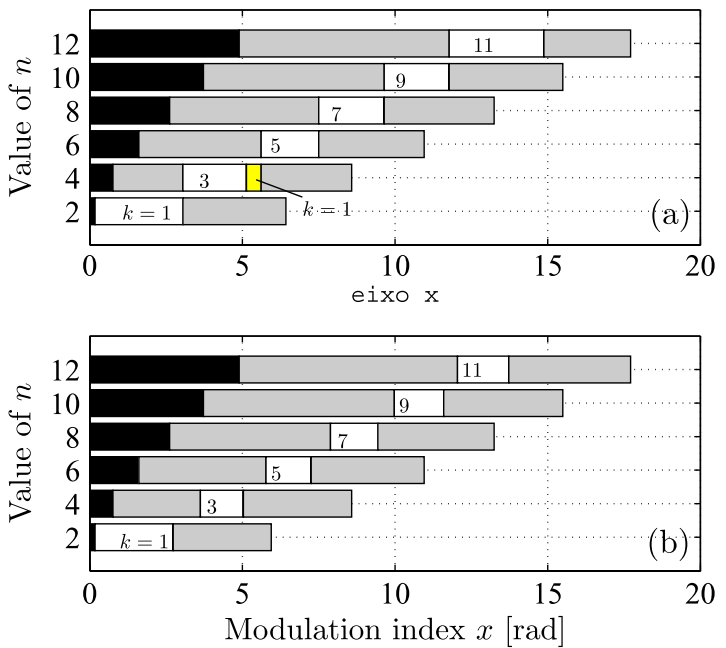

Fig. 6. Information relative to dynamic range (gray strips), envelope of maxima (white strips), and large error (black strips) for each value of $k$ (odd). (a) $\phi_{0}(t)$ between $\pi / 2 \pm 0.3156 \pi \mathrm{rad}$ and between $3 \pi / 2 \pm 0.3156 \pi \mathrm{rad}$. (b) $\phi_{0}(t)=0.2641 \pi \mathrm{rad}$. Ambiguity case $k=1$ is shown in yellow strip. 
$\left.V_{n}(x)\right]$. The regions in black correspond to large errors, due to noise. As can be seen, the white strips are accommodated loosely inside the low-error gray strips and far away from the large-error black strips. A detailed study of the Bessel functions leads to the conclusion that, in the white strips intervals in Fig. 6(a), the functions $J_{n-1}(x), J_{n+1}(x)$, and $J_{n+3}(x)$ used in Eq. (3) have the same algebraic sign. This means that it is not necessary to use any sign correction algorithm, as occurs with the modified $J_{1} \ldots J_{4}$ method. This is valid for any value of $x$ larger than the MDPS, which was verified in this work up to $x=100 \pi \mathrm{rad}$. Then the choice of $n=k+1$ is adequate and will result in low-estimation errors for $x$. This will not be the case only in the lower limit of the dynamic range of $x$ for $k=1$ (or $n=2$ ), which is adjacent to a black strip, resulting in a limited resolution given by the MDPS. The yellow strip for $n=4$ in Fig. 6(a), which is adjacent to the white strip for $k=3$, corresponds to the ambiguity case (see Subsection 4.D). As only the odd harmonics are used in Eq. (4), in this $\phi_{0}(t)$ interval, there is only the term $P=\sin \phi_{0}(t)$; there is no term $Q=\cos \phi_{0}(t)$ and consequently no problems when $\phi_{0}(t)=p \pi / 2 \mathrm{rad}$, $p= \pm 1, \pm 3, \ldots$. Obviously, the values of $\phi_{0}(t)$ for $p=$ $0, \pm 2, \pm 4 \ldots$ do not cause any problems because they are out of the range considered in this subsection.

B. Range of $\phi_{0}(\mathrm{t})$ between $0 \pm 0.1974 \pi \mathrm{rad}$ and between $\pi \pm 0.1974 \pi \mathrm{rad}$

In this range, which can be identified (first quadrant) in Fig. 5 , the even harmonics are higher than the odd ones because the modulus of factor $Q=\cos \phi_{0}(t)$ is close to one, except in the interval $x \leq 2.205 \mathrm{rad}$, where the first harmonic $(k=1)$ may be larger. For a fixed value of $\phi_{0}(t)$ and increasing values of $x$, the harmonic with order $k=1$ is larger, then $k=2$, then $k=4$, then again $k=2$ (another ambiguity case, for $6.3816<x<6.6417 \mathrm{rad}$, which will be treated later), then $k=6$, and so on. Furthermore, except for the ranges for $k=1$ and $k=2$ (the first one), all the other ranges have fixed width in $x$, independently of $\phi_{0}(t)$. Therefore, by increasing $\phi_{0}(t)$, the values of $x$ when $\left|V_{1}\right|$ is maximum stretch within the range $0 \leq x_{V_{1}} \leq 2.205 \mathrm{rad}$. Moreover, the values of $x$ where $\left|V_{2}\right|$ is maximum for the first time span within the $\left(x_{V_{1}}, 4.2011\right) \mathrm{rad}$ range. When $\phi_{0}(t)=0 \mathrm{rad}$, the $V_{1}$ component vanishes [as $P=\sin \phi_{0}(t)$ becomes null], existing as only maximum $\left|V_{k}\right|$ for even values of $k$.

As represented in Fig. 7(a), the ranges of $x$ where the envelope of a determined harmonic $k$ is larger are represented by white strips. In the particular case for $k=2(n=3)$ the maximum possible range (up to $x=4.2011 \mathrm{rad}$ ) was used, and the case $k=1$ will be discussed below. As before, the dynamic ranges obtained from Table 1 are represented by gray strips, and the regions with intolerable errors are represented by black strips. As can be seen, each white strip accommodates loosely in its corresponding dynamic range, and then there will be a negligible error in the estimation of $x$ if that value of $k$ (or $n$ ) is used.
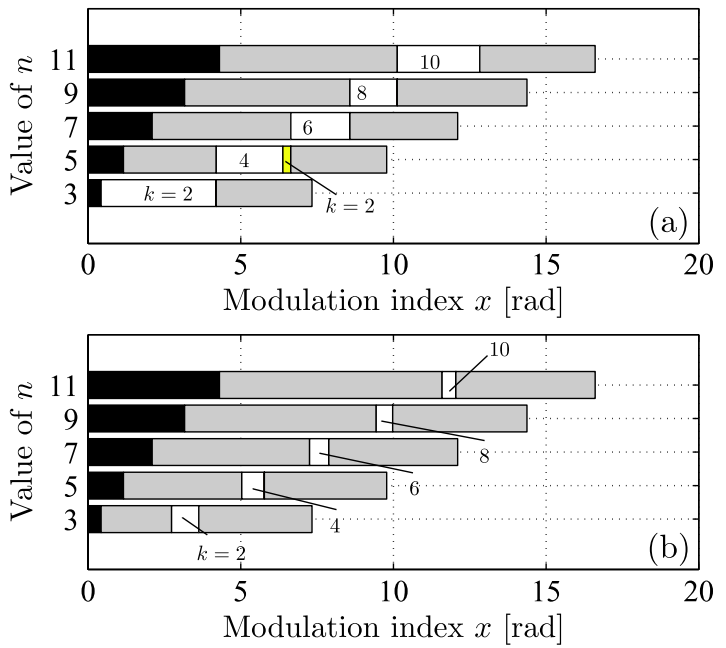

Fig. 7. Information relative to dynamic range (gray strips), envelope of maxima (white strips), and large error (black strips) for each value of $k$ (even). (a) $\phi_{0}(t)$ between $0 \pm 0.1974 \pi \mathrm{rad}$ and between $\pi \pm 0.1974 \pi \mathrm{rad}$. (b) $\phi_{0}(t)=0.2641 \pi \mathrm{rad}$. Ambiguity case $k=2$ is shown in yellow strip.

When the first harmonic is higher, a new white strip for $k=1$ in Fig. 6(a) could be considered, for $x$ between the MDPS $(x=0.1790 \mathrm{rad})$ and $2.205 \mathrm{rad}$, and thus it is a subset of the first one. This range could also be accommodated in the dynamic range for $n=2(k=1)$ in Table 1 , so it was not plotted in Fig. 7(a).

In the white strip ranges, the Bessel functions $J_{n-1}(x), J_{n+1}(x)$, and $J_{n+3}(x)$, used in Eq. (3), have the same algebraic sign, and no sign correction algorithm is necessary. Consequently, in this range of $\phi_{0}(t)$, only the harmonic with $k=1$ or with even orders can be maximum. By first considering the case of even $k$, only the factor $Q=\cos \phi_{0}(t)$ is used in Eq. (4), and, as the factor $P=\sin \phi_{0}(t)$ is not used, there will be no singularity problems when $\phi_{0}(t)=p \pi \mathrm{rad}, p= \pm 1, \pm 3, \ldots$. When $k=1$, the situation fits perfectly to the case of Subsection 4.A, being valid all considerations made there, regarding singularities and change of algebraic sign. Consequently, the $n=k+1$ choice is again adequate. The ambiguity case when $k=2$ [yellow strip adjacent to the white strip for $k=4$ in Fig. 7(a)] will be discussed in Subsection 4.D.

\section{Remaining Values of $\phi_{0}(\mathrm{t})$}

In this subsection, the remaining intervals of $\phi_{0}(t)$ will be analyzed, and it will be shown that the case considered here is even less restrictive than the ones described in Subsections 4.A and 4.B.

As observed in Fig. $\underline{5}$, for $\phi_{0}(t)=\overline{\pi / 2} \mathrm{rad}$, for example, the odd harmonics are maxima. When the value of the modulation index is increased, the first harmonic $\left|V_{1}\right|$ becomes maximum for the first time for $x$ between 0 and $3.0558 \mathrm{rad}$, and then a second time for $x$ between 5.1363 and 5.6164 rad. Considering now $\phi_{0}(t)=0.2641 \pi \mathrm{rad}$ in Fig. 5 , the region where $\left|V_{1}\right|$ becomes maximum for the second time 
disappears because it is surpassed by $\left|V_{4}\right|$. It is also evident that, unlike the previous cases examined in Subsections 4.A and 4.B, both even and odd harmonics can now assume maximum values. An interesting fact to be noticed is that, within this remaining range of $\phi_{0}(t)$, if the largest harmonic is odd (for a given $x$ ), the extent of its strip is now less than or equal to that of Fig. 6(a). In Fig. 6(b), the white strips correspond to the new range of maximum for odd $k$ and the particular case where $\phi_{0}(t)=0.2641 \pi \mathrm{rad}$. Now each new white strip is a subset of the corresponding strip that was shown in Fig. 6(a), and therefore all their properties are the same: it accommodates itself loosely inside each dynamic range associated with the index $n$ (established in Table 1), presents no problems with singularities when $\phi_{0}(\bar{t})=\pi / 2$ or $\pi \mathrm{rad}$, and requires no algorithms for algebraic sign corrections.

In summary, inside this complementary range of $\phi_{0}(t)$, if $k$ is associated with a maximum magnitude, the choice $n=k+1$ is again adequate. Moreover, this case is less restrictive than the one analyzed in Subsection 4.A because each white strip in Fig. 6(b) accommodates inside the corresponding white strip in Fig. 6(a). The same can be concluded when the maximum corresponds to harmonic $\left|V_{k}\right|$ for even $k$. If $\phi_{0}(t)=0 \mathrm{rad}$ in Fig. $\underline{5}$, the even harmonics are maxima, and $\left|V_{2}\right|$ becomes maximum for the second time in the range of $x$ between 6.3816 and $6.6417 \mathrm{rad}$. Using again the case where $\phi_{0}(t)=0.2641 \pi \mathrm{rad}$ as an example, Fig. 5 reveals that the region where the second harmonic $(k=2)$ becomes maximum for the second time disappears, being exceeded by component $\left|V_{5}\right|$. Furthermore, if the highest harmonic for a given $x$ is even, the extent of its strip is also less than or equal to those in Fig. 7(a), as shown in Fig. $7(\mathrm{~b})$ for $\phi_{0}(t)=0.2641 \pi \mathrm{rad}$. Again, as each new white strip in Fig. 7(b) is a subset of the corresponding strips that were shown in Fig. 7(a), they retain all their properties (is accommodated loosely inside each dynamic range, presents no problems with singularities when $\phi_{0}(t)=\pi / 2$ or $\pi \mathrm{rad}$, and requires no algorithms for algebraic sign corrections).

D. Ambiguity Cases for $\mathrm{k}=1$ and $\mathrm{k}=2$

The ambiguity cases occur when, as $x$ increases, the harmonic with $k=1$ or $k=2$ becomes maximum for the second time. This constitutes a problem just because it is more adequate to work only with harmonic magnitudes, without considering the phases. For $k=1$ and referring to the interval of $\phi_{0}(t)$ analyzed in Subsection 4.A, this range comprises the interval $5.1363<x<5.6164 \mathrm{rad}$. A detailed analysis of the magnitudes given by Eq. (2a) leads to the conclusion that if the harmonic with maximum envelope has order $k=1$, and if the ratio $\left|V_{5} / V_{1}\right|<0.6$, for the first time, then $x$ will be between 0 and $3.5057 \mathrm{rad}$, which comprises the range where $\left|V_{1}(x)\right|$ is maximum for the first time. Consequently, the choice $n=k+1$ can still be applied, and the value $n=2$ should be used in Eq. (4). On the other hand, if $\left|V_{5} / V_{1}\right| \geq 0.6$, for the first time, then $x$ will be between 3.5057 and $8.1493 \mathrm{rad}$, which comprises the range where $\left|V_{k}\right|$, for $k=1$, is maximum for the second time. Again, the value $n=2$ could be used, and a second white strip could be accommodated within the gray strip (region of small errors) for $n=2$ in Fig. 6(a). However, it would be necessary to apply a sign correction algorithm for $V_{1}$ because it becomes negative in this gray strip region for $n=2$. On the other hand, if this second strip (now in yellow) is accommodated within the gray strip for $n=4$, there is no need to sign correction. Consequently, the value $n=4$ should be used in Eq. (4).

The ambiguity case for $k=2$ is treated in a similar way: if, in the range of $\phi_{0}(t)$ considered in Subsection 4.B, the harmonic with maximum magnitude has order $k=2$, and if $\left|V_{6} / V_{2}\right|<0.6$ for the first time, then $x$ will be between 0 and $4.6615 \mathrm{rad}$. Then the practical rule $n=k+1$ is followed and $n=3$ is used in Eq. (4). Otherwise, if $\left|V_{6} / V_{2}\right| \geq 0.6$ for the first time, corresponding to $4.6615<x<9.3745 \mathrm{rad}$ (which comprises the range where $\left|V_{2}\right|$ is maximum for the second time), then the choice $n=5$ is suggested in Eq. (4), related to the yellow strip in Fig. 7(a), where it is not necessary to use any algorithm of algebraic sign correction.

In both ambiguity cases, there will be no problems with singularities of the type $\phi_{0}(t)=p \pi / 2 \mathrm{rad}$, $p= \pm 1, \pm 2, \ldots$ According to Fig. 5 , for the range of $\phi_{0}(t)$ discussed in Subsection 4. $\overline{\mathrm{C}}$, the regions for $k=1$ and $k=2$ for the second time become subsets of those shown in Figs. 6(a) and 7(a) (and hence are treated the same way) or disappear (consequently, there is no need to concern).

\section{E. Simulations Using the $n$-CPM}

By considering $0 \leq x \leq 15 \mathrm{rad}, 0 \leq \phi_{0}(t) \leq 2 \pi \mathrm{rad}$, $m=1$, and $K=0.0011$, the relative percent error $\Delta x_{r}=100\left|x^{\prime}-x\right| / x$ is numerically calculated in Matlab using the $n$-CPM. The result is shown in Fig. 8, where the error was truncated to $10 \%$ for $x$ close to zero. As can be observed, the bathtubs present in the original method disappear, showing the efficiency of the proposed method.

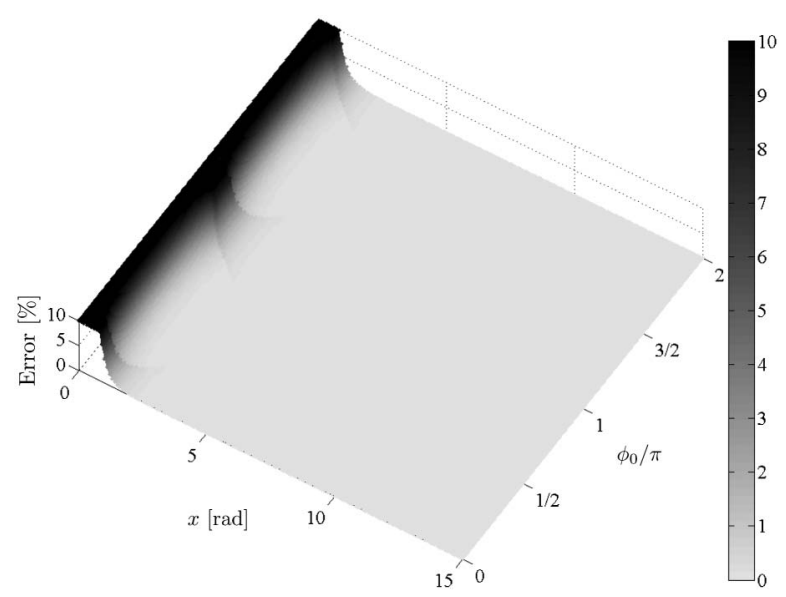

Fig. 8. Bathtub graph for the $n$-CPM. 
In the following, Eq. (4) was simulated by considering $-46 \mathrm{dBV}$ additive white Gaussian noise and a value of $\phi_{0}(t)$ varying randomly between 0 and $\pi / 2 \mathrm{rad}$. This kind of noise (for $m=0$ ) fits more to the real case found in practical experiments in the present work. The result is shown in Fig. 9, in terms of the estimated value of the modulation index $x^{\prime}$ as a function of $x$ (in circles). Some values of $n$ used at each point are represented in the figure, illustrating how the algorithm based on the proposition selects the best value of $n$ for the estimation of $x$. The straight line corresponds to the curve $x^{\prime}(x)=x$. The value of $\phi_{0}(t)$ used in the simulation is also plotted (triangles, right vertical axis). When $\phi_{0}(t) \sim 0$, the even harmonics have larger magnitudes, and an odd value of $n$ is selected. When $\phi_{0}(t) \sim \pi / 2 \mathrm{rad}$, the odd harmonics have larger magnitudes, and an even value of $n$ is selected. The error between $x^{\prime}$ and $x$ is very small (less than $0.07 \%$ ).

\section{Experimental Results}

A He-Ne laser (Newport, $15 \mathrm{~mW}, \lambda=632.8 \mathrm{~nm}$ ) is used as an optical source in an open-loop homodyne Michelson interferometer. The laser is divided by a $50 / 50$ beam splitter in the sensor and reference arms of the interferometer. The sensor arm impinges on a slender mirror (reflective tape), which is glued to the $\mathrm{XY}$ nanopositioner shown in Fig. 1 , while the reference arm impinges on a fixed mirror. Precision micrometric stages for translation, rotation, and tilt are used to interferometer alignment, so that the rays reflected from the mirrors return to the beam splitter and are superimposed at the active area of a photodiode (ThorLabs, PDA 55 Amplified Si Photodetector, $10 \mathrm{MHz}$ bandwidth, 400-1100 $\mathrm{nm}$ range). In the experiments, the interferometric signal $v(t)$ is digitized by an oscilloscope (Tektronix TDS 2022), and each acquisition window has 2500 samples. The data is transferred to a computer and processed in Matlab.

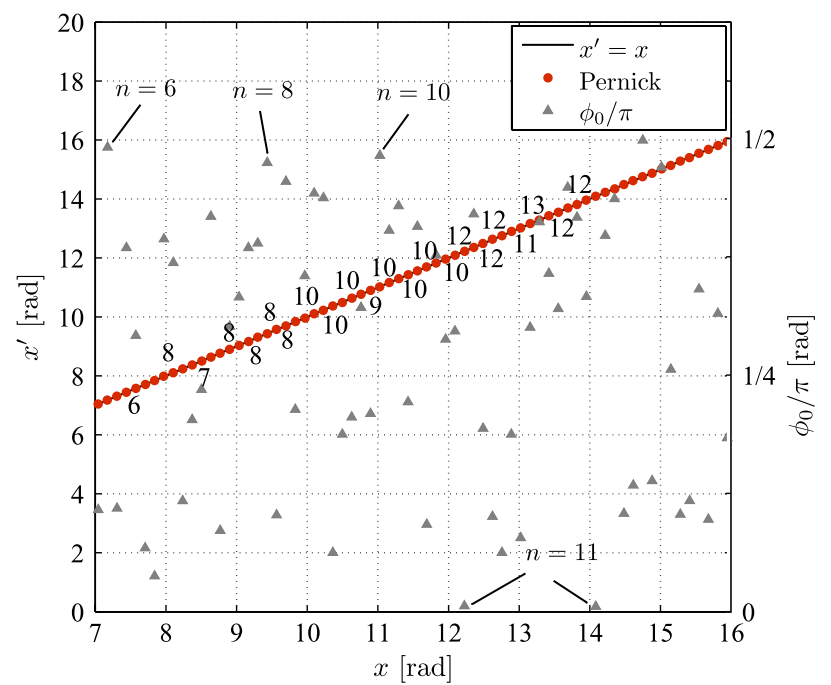

Fig. 9. Simulation of the $n$-CPM showing the selection of the best values of $n$ for some points.
The signals are Hamming-windowed and zeropadded before spectral computation. The sampling rate is adjusted, so that there are at least 10 signal periods per acquisition window in order to result in a good spectral resolution. On the other hand, the acquisition window should not be too long because, if $\phi_{0}(t)$ has a significant drift in this interval, $v(t)$ is not periodic, and the series expansion in Eq. (1) cannot be applied.

The XY nanopositioner was driven by a sinusoidal voltage by using a function generator (Agilent 22330A) and a power amplifier (A. A. Lab Systems, A-301HS Piezo Amplifier), reaching voltages up to $350 \mathrm{~V}$ (peak-to-peak) for frequencies smaller than $1 \mathrm{kHz}$. Laboratory measurements showed that white noise was predominant, with $-61.5 \mathrm{dBV}$, approximately. The modulation index $x$ was then calculated using the $n$-CPM, and the maximum output displacement is obtained from $\Delta L=x \lambda / 4 \pi$, where $\lambda$ is the laser wavelength.

The XY nanopositioner is an MAPFA with high mechanical flexibility for generated and coupled movements. Due to the flexibility, large dynamic displacements can be generated in the structure, even using low-input voltages. The generated movement displacements can be observed in Fig. 10 for sinusoidal input voltages from 0 to $160 \mathrm{~V}$ (peak) at the frequencies of 370 and $930 \mathrm{~Hz}$. Each point in the curve (and in the other results) corresponds to the mean obtained from 10 measurements. There is a linear response starting from $0.2 \mathrm{rad}$, and the MAPFA linear length-to-voltage sensitivities resulted in 13.4 and $20.4 \mathrm{~nm} / \mathrm{V}$ (peak), at the frequencies of 370 and $930 \mathrm{~Hz}$, respectively.

The cross-movement linearity response is shown in Fig. 11 for voltages between 0 and $52 \mathrm{~V}$ (peak) at the frequencies of 290 and $910 \mathrm{~Hz}$. The coupled movement also shows a linear response, with linear length-to-voltage sensitivities of 2.95 and $11.1 \mathrm{~nm} / \mathrm{V}$

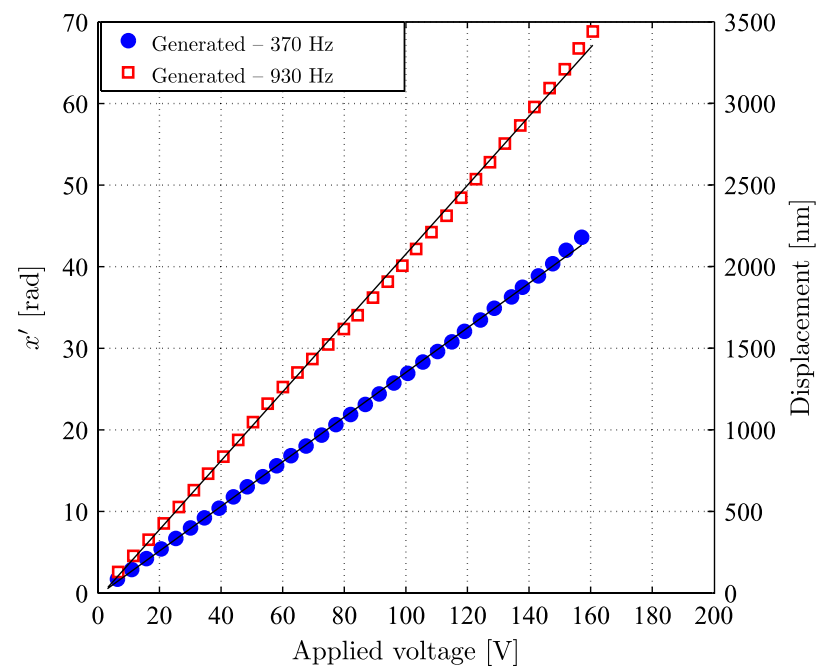

Fig. 10. XY nanopositioner generated movement linearity curves at the frequencies of 370 and $930 \mathrm{~Hz}$. 


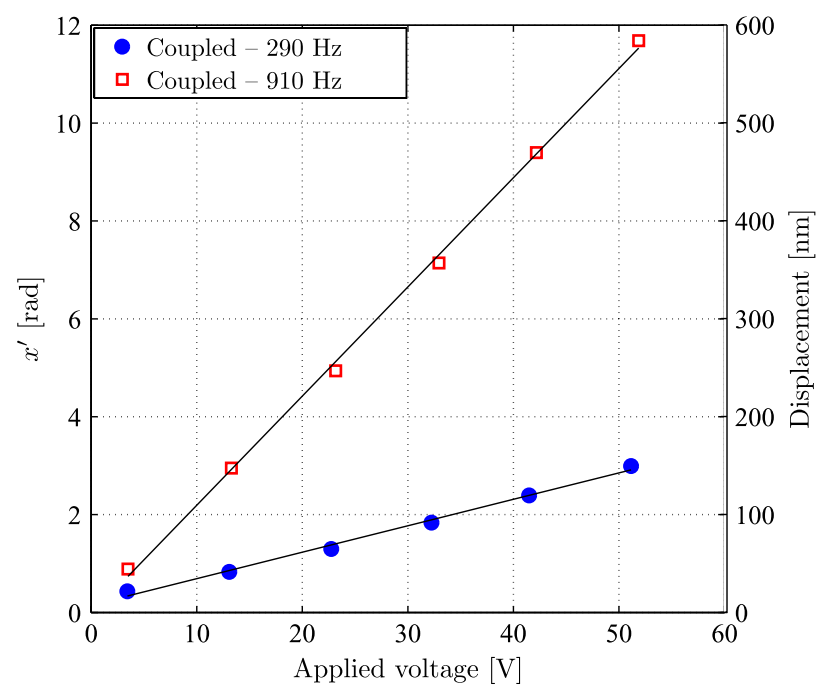

Fig. 11. XY nanopositioner coupled movement linearity curves at the frequencies of 290 and $910 \mathrm{~Hz}$.

(peak) at the frequencies of 290 and $910 \mathrm{~Hz}$, respectively. The coupled movements measured in this prototype have significant amplitudes that are larger than desired, which could demand a redesign of the device.

The frequency response shows the MAPFA mechanical resonance frequencies as well as the quasi-static range of operation. The MAPFA generated and coupled movements frequency response, in terms of the displacement-to-applied voltage ratio, is shown in Fig. 12, for frequencies between 90 and $1270 \mathrm{~Hz}$. Each point in the frequency response curve is the slope obtained from a linearity curve. There are resonances around 230,390, 990, 1190, and $1230 \mathrm{~Hz}$. As observed in Fig. 12, at these frequencies the coupled displacement have similar magnitudes as the generated movements, which is a nonintuitive result.

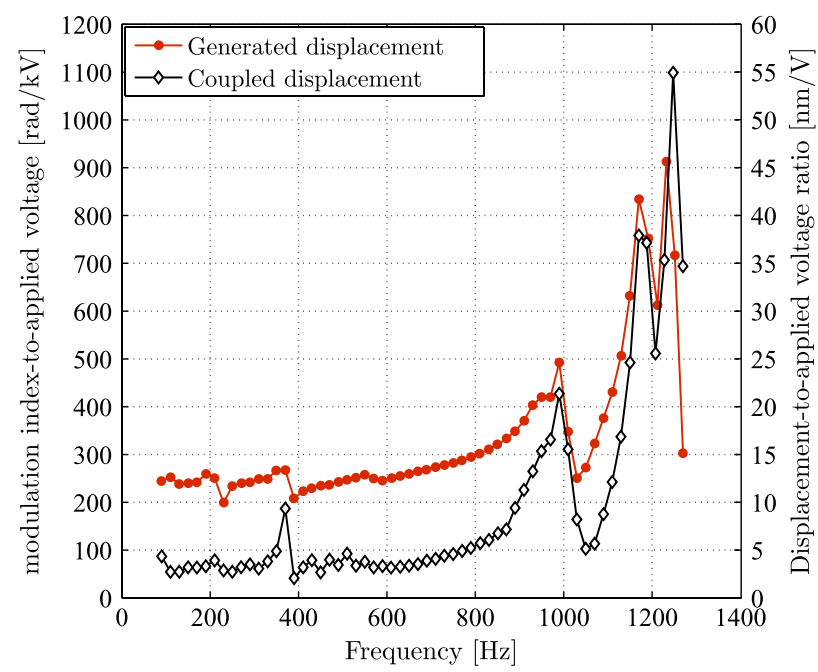

Fig. 12. Generated and coupled XY nanopositioner displacement frequency response, in terms of modulation index- and displacement-to-applied voltage ratio.

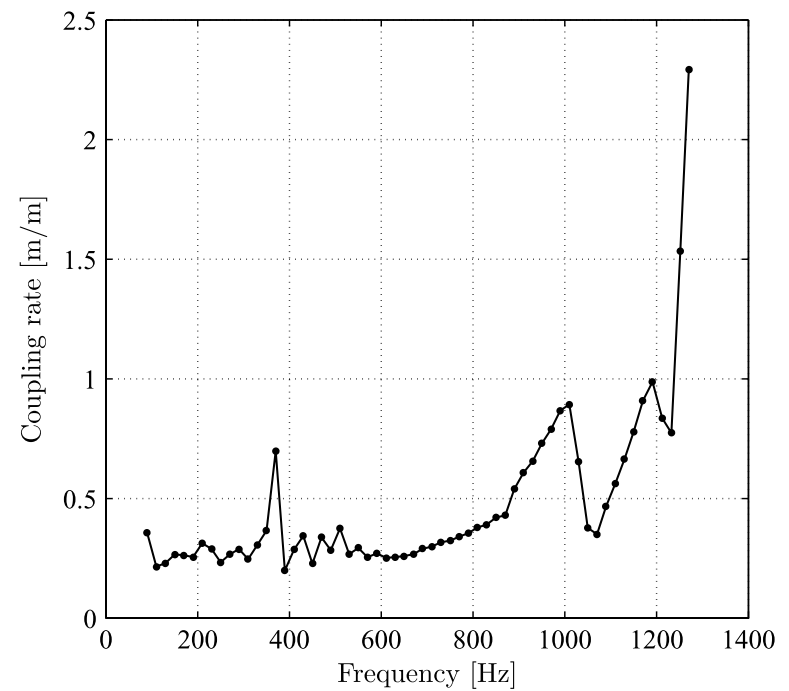

Fig. 13. Coupling rate $S_{x y}$ as a function of frequency.

From the data of generated and coupled movements (Fig. 12), the coupling rate $S_{x y}$ can be measured as a function of frequency. The result is shown in Fig. 13. As a general result, this XY nanopositioner should operate below $200 \mathrm{~Hz}$ approximately, far away from mechanical resonances. Furthermore, in this frequency band the coupled displacements are around $25 \%$, which is larger than desired. The difference between the computational simulations and prototype may be related to manufacturing issues such as the bonding of the piezoceramics to the metal structure [23]. A thick bonding layer may compromise the displacement transmission from the piezoceramic to the metallic structure. This will require an improvement of the manufacturing process.

\section{Conclusions}

In this article, some classical methods based on homodyne interferometry and the photodetected signal spectrum were mentioned, but that have problems related to resolution, reduced dynamic range, and singularities close to the condition $\phi_{0}(t)=$ $p \pi / 2 \mathrm{rad}, p=1,2,3, \ldots$, where $\phi_{0}(t)$ is a random signal. In order to overcome most of these limitations, this work proposed to use the recurrence relation in Eq. (3) and create a new phase-demodulation technique by adding to it an algorithm used to choose an adequate value of $n$ in Eq. (4). The proposed $n$-CPM is homodyne, passive, direct, self-consistent, without phase ambiguity problems, immune to fading, does not demand algebraic signal correction and has a large dynamic range.

As limitations, the $n$-CPM has relatively poor resolution, measuring modulation indexes larger than $0.2 \mathrm{rad}$ (or $10 \mathrm{~nm}$ for the laser used), operates only with low-distortion sinusoidal signals, and does not allow the characterization of nonlinear devices. Actually, in a nonlinear regime, there is no direct relationship between the applied voltage and output displacement waveforms and neither with the 
waveform of the interferometer-induced optical phase shift. Consequently, the periodic signal requirements for the Fourier series expansion in Eq. (1) is no longer satisfied. However, this characteristic is intrinsic to all conventional methods based on the signal spectrum analysis discussed in the text.

Simulations considering $1 / f$ voltage additive noise revealed that the $n$-CPM presents a maximum percent error of only $1 \%(3 \%)$ in the $0.6 \mathrm{rad}(0.36 \mathrm{rad})$ to $100 \pi \mathrm{rad}$ modulation index range. In this dynamic range, the method eliminates all imperfections in the bathtubs graphs of the original Pernick method, which occur due to $0 / 0$ and $\phi_{0}(t)=p \pi / 2 \mathrm{rad}$ singularities.

Large displacements can be measured with the $n$-CPM, as those generated by the XY nanopositioner shown in Fig. 1, whose flexibility is very high. This device has a bandwidth smaller than $200 \mathrm{~Hz}$ and multiple resonances above this frequency. When the actuator operates in resonance, the whole structure vibrates, and the generated and coupled displacements are of the same order of magnitude. The coupling factor was calculated, revealing its frequency dependence.

The authors are grateful to the research agencies FAPESP (project number 2012/01629-7), CNPq (project number 303689/2009-9 and 478817/2012-6) and CAPES in Brazil.

\section{References}

1. S. Devasia, E. Eleftheriou, and S. Moheimani, "A survey of control issues in nanopositioning," IEEE Trans. Control Syst. Technol. 15, 802-823 (2007).

2. S. T. Smith and D. G. Chetwynd, "Foundations of ultraprecision mechanism design," in Developments in Nanotechnology (Gordon and Breach Science, 1992), Vol. 2, pp. 95-180.

3. R. C. Carbonari, E. C. N. Silva, and S. Nishiwaki, "Optimum placement of piezoelectric material in piezoactuator design," Smart Mater. Struc. 16, 207-220 (2007).

4. R. C. Carbonari, E. C. N. Silva, and S. Nishiwaki, "Design of piezoelectric multi-actuated microtools using topology optimization," Smart Mater. Struc. 14, 1431-1447 (2005).

5. Y. Tian, B. Shirinzadeh, and D. Zhang, "A flexure-based five-bar mechanism for micro/nano manipulation," Sens. Actuators A 153, 96-104 (2009).

6. O. M. E. Rifai and K. Youcef-Toumi, "Trade-offs and performance limitations in mechatronic systems: a case study," Ann. Rev. Cont. 28, 181-192 (2004).

7. F. Claeyssen, R. Le Letty, F. Barillot, N. Lhermet, H. Fabbro, P. Guay, M. Yorck, and P. Bouchilloux, "Mechanisms based on piezoactuators," Proc. SPIE 4332, 225-233 (2001).

8. A. Eisinberg, A. Menciassi, S. Micera, D. Campolo, M. Carrozza, and P. Dario, "Pi force control of a microgripper for assembling biomedical microdevices," IEE Proc. Circuits Devices Syst. 148, 348-352 (2001).
9. A. Menciassi, A. Eisinberg, M. Carrozza, and P. Dario, "Force sensing microinstrument for measuring tissue properties and pulse in microsurgery," IEEE/ASME Trans. Mechatron. 8, 10-17 (2003).

10. E. Udd and W. B. J. Spillman, Fiber Optic Sensors: An Introduction for Engineers and Scientists (Wiley, 2011).

11. S. K. Sheem, T. G. Giallorenzi, and K. Koo, "Optical techniques to solve the signal fading problem in fiber interferometers," Appl. Opt. 21, 689-693 (1982).

12. F. Xie, J. Ren, Z. Chen, and Q. Feng, "Vibration-displacement measurements with a highly stabilised optical fiber Michelson interferometer system," Opt. Laser Technol. 42, 208-213 (2010).

13. S. Yokoyama, T. Yokoyama, and T. Araki, "High-speed subnanometre interferometry using an improved threemode heterodyne interferometer," Meas. Sci. Technol. 16, 1841-1847 (2005).

14. S. Zhen, B. Chen, L. Yuan, M. Li, J. Liang, and B. Yu, "A novel interferometric vibration measurement sensor with quadrature detection based on 1/8 waveplate," Opt. Laser Technol. 42, 362-365 (2010).

15. S. Kang, J. La, H. Yoon, and K. Park, "A synthetic heterodyne interferometer for small amplitude of vibration measurement," Rev. Sci. Instrum. 79, 053106 (2008).

16. D. T. Smith, J. R. Pratt, and L. P. Howard, "A fiber-optic interferometer with subpicometer resolution for dc and lowfrequency displacement measurement," Rev. Sci. Instrum. 80, 035105 (2009).

17. S. Topcu, L. Chassagne, Y. Alayli, and P. Juncar, "Improving the accuracy of homodyne Michelson interferometers using polarisation state measurement techniques," Opt. Commun. 247, 133-139 (2005).

18. V. S. Sudarshanam and K. Srinivasan, "Linear readout of dynamic phase change in a fiber-optic homodyne interferometer," Opt. Lett. 14, 140-142 (1989).

19. V. Sudarshanam and R. Claus, "Generic J1...J4 method of optical phase detection,” J. Mod. Opt. 40, 483-492 (1993).

20. B. J. Pernick, "Self-consistent and direct reading laser homodyne measurement technique," Appl. Opt. 12, 607-610 (1973).

21. H. A. Deferrari, R. A. Darby, and F. A. Andrews, "Vibrational displacement and mode-shape measurement by a laser interferometer," J. Acoust. Soc. Am. 42, 982-990 (1967).

22. W. Jin, L. M. Zhang, D. Uttamchandani, and B. Cuishaw, "Modified J1...J4 method for linear readout of dynamic phase changes in a fiber-optic homodyne interferometer," Appl. Opt. 30, 4496-4499 (1991).

23. R. C. Carbonari, G. Nader, S. Nishiwaki, and E. C. N. Silva, "Experimental and numerical characterization of multiactuated piezoelectric device designs using topology optimization," in Proc. SPIE 5764, 472-481 (2005).

24. Y. Bitou, "High-accuracy displacement metrology and control using a dual Fabry-Perot cavity with an optical frequency comb generator," Precis. Eng. 33, 187-193 (2009).

25. M. M. Brundavanam, N. K. Viswanathan, and D. N. Rao, "Nanodisplacement measurement using spectral shifts in a white-light interferometer," Appl. Opt. 47, 6334-6339 (2008).

26. G. Heinzel, F. G. Cervanyes, A. F. G. Marin, J. Kullmann, W. Feng, and K. Danzmann, "Deep phase modulation interferometry," Opt. Express 18, 19076-19086 (2010).

27. V. S. Sudarshanam, "Minimum detectable phase shift in spectrum-analysis techniques of optical interferometric vibration detection,” Appl. Opt. 31, 5997-6002 (1992). 